Research Article

\title{
Prediction of Frequency Response Function for Cylindrical Thin-Walled Workpiece with Fixture Support Constraints
}

\author{
Jinjie Jia (D), Yuwen Sun (D), and Jinbo Niu $\mathbb{D}$ \\ Key Laboratory for Precision and Non-Traditional Machining Technology of Ministry of Education, \\ School of Mechanical Engineering, Dalian University of Technology, Dalian 116024, China
}

Correspondence should be addressed to Jinbo Niu; niujinbo@hotmail.com

Received 14 March 2021; Revised 20 April 2021; Accepted 30 July 2021; Published 14 August 2021

Academic Editor: Alberto Campagnolo

Copyright $(92021$ Jinjie Jia et al. This is an open access article distributed under the Creative Commons Attribution License, which permits unrestricted use, distribution, and reproduction in any medium, provided the original work is properly cited.

Auxiliary fixtures are widely used to enhance the rigidity of cylindrical thin-walled workpieces (CTWWs) in the machining process. Nevertheless, the accurate and efficient prediction of frequency response function (FRF) for the workpiece-fixture system remains challenging due to the complicated contact constraints between workpiece and fixture. This paper proposes an analytical solution for the comprehensive FRF analysis of the CTWW-fixture system. Firstly, based on the vector mechanics, the mode shape functions of the workpiece are presented using the classical theory of thin shell. The variable separation method is utilized to deal with the inter-mode coupling of the workpiece. Secondly, the motion equation of the CTWW with fixture constraints is established using analytical mechanics from the viewpoint of energy balance. Finally, the FRFs of the CTWW-fixture system are derived by means of modal superposition. Experimental modal tests verify that the predicted FRFs are in good agreement with the measured curves.

\section{Introduction}

Cylindrical thin-walled parts such as engine casings are widely used in aerospace industries. Due to the low rigidity of thin-walled workpiece, it is prone to produce large-amplitude vibrations and even regenerative chatter during the machining process $[1,2]$, which consequently affect the final part quality $[3,4]$. In order to enhance the rigidity of the machining system, auxiliary fixtures have been widely designed and used to enhance the rigidity of thin-walled parts [5].

Positioning error and clamping stability under static or quasistatic loads are primary concerns with regard to fixture design. There are two main sources of positioning errors: the contact compliance between the workpiece and the fixture [6] and the geometric errors of the locators [7, 8]. The positioning accuracy is affected by many factors including dimensional error $[9,10]$, clamping force [11], clamping sequence [12], and so on. The clamping stability is affected not only by the contact compliance but also by the rigidity of the fixture components [13]. To determine the stability, the screw theory [14] is widely used, where the applied load is equivalently regarded as a rotation and a translation along the same axis.

For flexible workpieces, the compliance cannot be neglected when analyzing the contact status between workpiece and fixture. As far as the positioning error is concerned, it is mainly affected by the local deformation of the workpiecefixture contact area. Also, the deformation contribution caused by the workpiece-fixture contact compliance is much smaller than the workpiece deformation. Thus, the workpiece-fixture contact could be regarded as rigid compared to the compliance of the workpiece [15]. Moreover, for analyzing the system stability, the difference between rigid workpieces and flexible workpieces lies in the influence of workpiece compliance on clamping force and contact. Among them, the minimum clamping force [16] needs to be determined to prevent slippage of the workpiece, and a reasonable contact area [17] needs to be identified to meet the form-closure condition. From the above analysis, the compliance of the workpiece should be taken into account, which significantly affects the quality of machined surfaces [18]. 
The above works regard the workpiece-fixture system as static or quasistatic and ignore the dynamic characteristics. Nevertheless, the interrupted milling forces have high-frequency components, especially for thin-walled workpieces [3]. Therefore, the performance of fixture should be evaluated under dynamic loads. Rigid body dynamics [19], as the basis of the dynamics research of the workpiece-fixture system, is widely used. Among them, the research studies of workpiece-fixture contact including contact modeling [20] and contact analysis [21] are very critical. Contact modeling is mainly studied by means of analytical methods [22-24] and finite element methods $[25,26]$. For contact analysis, many factors such as nonlinear behavior [27], stiffness [28], friction damping [29], and so on should be considered, which have great influence on the dynamics of rigid bodies. In this way, the fixturing stability of the system [30] and the optimal design of the fixture [19] can be effectively analyzed.

Regarding the influence of fixtures on the dynamics of flexible workpieces, however, the relevant research is very limited. Due to the weak rigidity of flexible workpieces, adding support [31, 32], which has a great impact on the dynamic characteristics of the workpiece, in the fixture design is an effective way. In order to study it, the frequency response function (FRF) [33] is often used for analysis. In the analysis process, the finite element method (FEM) $[34,35]$ is an excellent numerical tool used to obtain relevant detailed information. When using this method, it was necessary to divide very fine grids and set complex equivalent boundary conditions to ensure the accuracy of the results, which would cause a significant reduction in calculation efficiency [36]. To solve this problem, the analytical method focusing on thinwalled plate or frame structures $[37,38]$ is widely used to analyze the effects of fixture on the dynamic characteristics of the workpiece.

Nevertheless, to the authors' knowledge, few research concentrates on the effects of fixtures on cylindrical thinwalled structures, especially from the perspective of dynamics. Under the motivation, the main objective of this paper is to develop a comprehensive analytical solution to predict the FRFs of the system so as to investigate the effects of fixture support on the dynamic characteristics of CTWW. The outline of this paper is organized as follows. Section 2 establishes the motion equation of the workpiece-fixture system. The frequency response functions of the workpiecefixture system are predicted in Section 3. Section 4 verifies the feasibility and accuracy of the proposed method. Some important conclusions are listed in Section 5.

\section{Motion Equation of the Workpiece- Fixture System}

Adding fixture support can effectively improve the rigidity of the CTWWs, which makes the modeling and analysis of the system more complicated. Therefore, it is necessary to focus on the establishment of the motion equation of the workpiece-fixture system, which is the basis for investigating the effects of fixture support on workpiece dynamic characteristics. Without losing generality, a typical thin-walled cylindrical structure with fixture constraints is taken to explore the mechanism, as shown in Figure 1. The outside surface of the cylindrical workpiece is required to be further milled from the free end to the fixed end for lightweight purposes. In this paper, the boundary conditions are determined according to the actual machining situation. The base of the workpiece is clamped, i.e., the slope and displacement are equal to zero. The top edge of the workpiece is free, i.e., the moments and shear forces are equal to zero.

2.1. Mode Shape Functions of the CTWW. In this section, the dynamic balance equation of the CTWW is established using the vector mechanics method. The mode shape functions can be obtained. The 3D model of this part can be equivalently represented using a continuous, homogeneous, and isotropous cylindrical shell, as shown in Figure 2.

The workpiece has an axial length $L$, a middle surface radius $R$, and a constant thickness $H$. A cylindrical coordinate system $(o, z, \theta, r)$ is used to take advantage of the axis symmetry of the structure, and the origin is set to the center of the fixed end $(z=0)$ of the workpiece. The displacements on the middle surface with respect to the coordinate system are represented by $u(z, \theta, t), v(z, \theta, t)$, and $w(z, \theta, t)$ in the tangential, radial, and axial directions, respectively. The CTWW is clamped at the bottom $(z=0)$, and the other end is free $(z=L)$. Since the thickness of the workpiece is much smaller than other dimensions, a thin-walled cylindrical shell assumption is considered. In addition, the straight line perpendicular to the middle surface of the workpiece remains unchanged before and after deformation [39].

In the modeling process, a series of cylindrical shell elements are used to simulate the middle surface. An arbitrary element is expressed in Figure 3. We take this element as an example to illustrate the modeling process. Each shell is constructed by an $\operatorname{arc} \operatorname{Rd} \theta$ and a line segment $\mathrm{d} z$, respectively. The forces including external forces $p_{e}(e=r, \theta, z)$ and inertial forces $\rho H\left(\partial \mathrm{in}^{2} / \partial t^{2}\right)($ in $=u, v, w)$ are shown in Figure 3(a), and the internal forces and internal moments are shown in Figures 3(b) and 3(c). By multiplying all internal forces by the arc length of the side, multiplying all external force components and inertial forces by the element area, and then projecting the product in three directions, the free vibration differential equations of the CTWW can be established as follows [40]:

$$
\left\{\begin{array}{l}
\frac{1}{R} \frac{\partial N_{\theta}}{\partial \theta}+\frac{\partial N_{z \theta}}{\partial z}+\frac{Q_{\theta}}{R}-\rho H \frac{\partial^{2} u}{\partial t^{2}}=0 \\
\frac{\partial Q_{z}}{\partial z}+\frac{\partial Q_{\theta}}{R \partial \theta}-\frac{N_{\theta}}{R}-\rho H \frac{\partial^{2} v}{\partial t^{2}}=0 \\
\frac{\partial N_{z}}{\partial z}+\frac{1}{R} \frac{\partial N_{z \theta}}{\partial \theta}-\rho H \frac{\partial^{2} w}{\partial t^{2}}=0
\end{array}\right.
$$

where $\rho$ is the density of the workpiece, $N_{\theta}, N_{z \theta}, N_{z}$ and $Q_{\theta}$, $Q_{z}$ are the internal forces and transverse shear forces per unit length on the middle surface, and their specific expressions are given by 


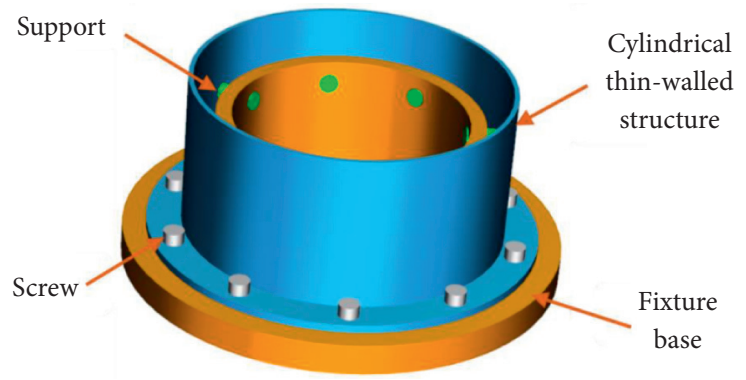

FIGURE 1: Schematic illustration of a typical cylindrical thin-walled structure with fixture constraints.

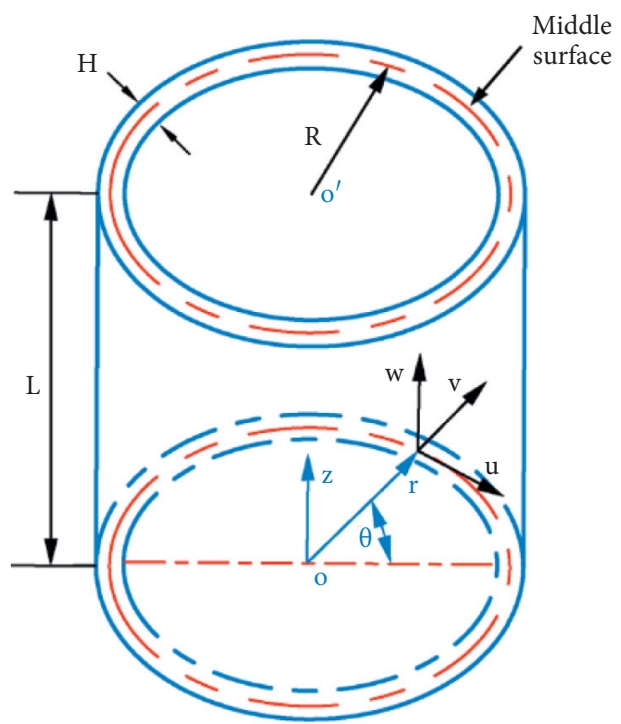

Figure 2: Coordinate system and dimensions for a cylindrical shell.
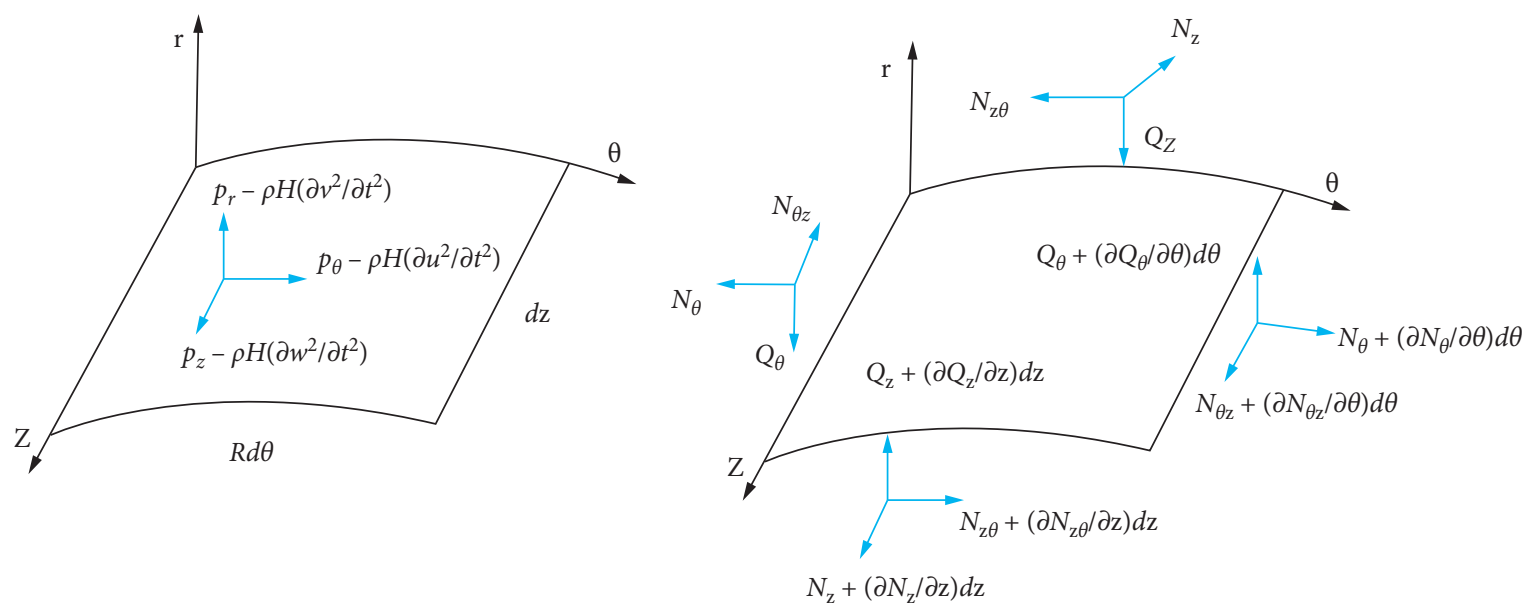

(a)

(b)

Figure 3: Continued. 


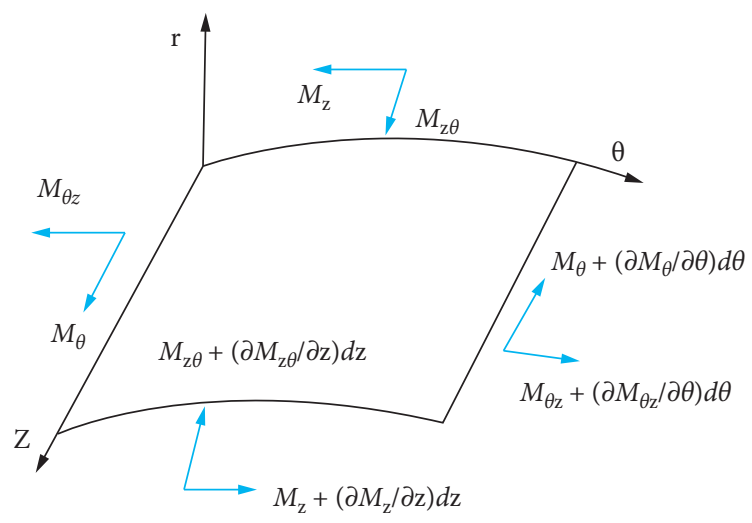

(c)

FIGURE 3: Forces and moments acting on a cylindrical shell element. (a) External forces and inertial forces. (b) Internal forces. (c) Internal moments.

$$
\left\{\begin{array}{l}
N_{\theta}=K\left[\frac{1}{R}\left(\frac{\partial u}{\partial \theta}+v\right)+\mu \frac{\partial w}{\partial z}\right] \\
N_{z \theta}=K \frac{1-\mu}{2}\left(\frac{\partial u}{\partial z}+\frac{1}{R} \frac{\partial w}{\partial \theta}\right) \\
N_{z}=K\left[\frac{\partial w}{\partial z}+\frac{\mu}{R}\left(\frac{\partial u}{\partial \theta}+v\right)\right] \\
M_{z}=D\left[-\frac{\partial^{2} v}{\partial z^{2}}+\frac{\mu}{R^{2}}\left(\frac{\partial u}{\partial \theta}-\frac{\partial^{2} v}{\partial \theta^{2}}\right)\right] \\
M_{\theta}=D\left[\frac{1}{R^{2}}\left(\frac{\partial u}{\partial \theta}-\frac{\partial^{2} v}{\partial \theta^{2}}\right)-\mu \frac{\partial^{2} v}{\partial z^{2}}\right] \\
Q_{z}=\frac{\partial M_{z \theta}}{\partial z}+\frac{\partial M_{\theta}}{R \partial \theta} \\
M_{z \theta}=D \frac{\partial M_{z \theta}}{2 R}, \mu\left(\frac{\partial u}{\partial z}-2 \frac{\partial^{2} v}{\partial z \partial \theta}\right) \\
Q_{\theta}
\end{array}\right.
$$

where $M_{z}, M_{\theta}$, and $M_{z \theta}$ are the internal moments on the middle surface, $K=E H /\left(1-\mu^{2}\right)$ is the tensile stiffness of the cylinder shell, $D=E H^{3} / 12\left(1-\mu^{2}\right)$ is the flexural rigidity of the cylindrical shell, $E$ is Young's modulus, and $\mu$ is Poisson's ratio.

By substituting equation (2) into (1), the governing equations expressed by displacements $u, v$, and $w$ can be obtained as

$$
\left[\begin{array}{lll}
L_{11} & L_{12} & L_{13} \\
L_{21} & L_{22} & L_{23} \\
L_{31} & L_{32} & L_{33}
\end{array}\right]\left\{\begin{array}{c}
u \\
v \\
w
\end{array}\right\}=\left\{\begin{array}{l}
0 \\
0 \\
0
\end{array}\right\},
$$

where $L_{i_{d} j_{d}}=\left(i_{d}, j_{d}=1,2,3\right)$ are differential operators with respect to $z$ and $\theta$, and the specific expressions are given in Appendix A.

To solve equation (3), a synchronous motion is considered, i.e., natural modes of vibration [41]:

$$
\left\{\begin{array}{l}
u(x, \theta, t)=U(x, \theta) q(t), \\
v(x, \theta, t)=V(x, \theta) q(t), \\
w(x, \theta, t)=W(x, \theta) q(t),
\end{array}\right.
$$

where $U(x, \theta), V(x, \theta)$, and $W(x, \theta)$ are the mode shapes and $q(t)$ is the generalized coordinate.

The vibration forms of CTWW include axial and circumferential vibrations, as shown in Figure 4. The axial vibration form in Figure 4(a) is composed of $m$ half-waves. The circumferential vibration form in Figure 4(b) consists of radial and tangential vibration, where $n$ is the number of circumferential waves. Therefore, the modal shape of the cylindrical shell can be determined by any combination of the axial half-wave number $m$ and the circumferential wave number $n$.

Considering the inter-mode coupling, a variable separation method is employed to separate the spatial dependence of modal shapes between axial and circumferential directions. The modal shape of the thin-walled cylindrical shell is expanded in a double series in terms of beam function in the axial direction and Fourier series in the circumferential direction. This method can use simple functions to decouple the complex coupled dynamics system and avoid the tedious mathematical calculation process. Therefore, the tangential, radial, and axial displacements of the workpiece vary according to [42] 

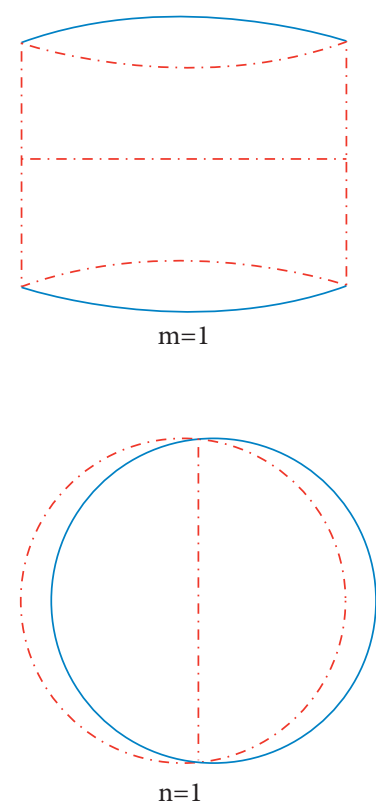

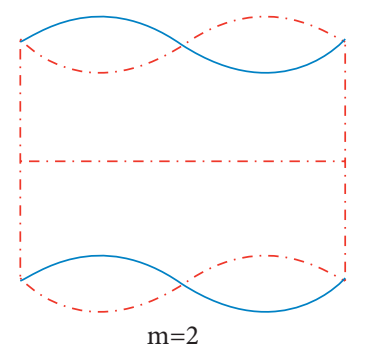

(a)

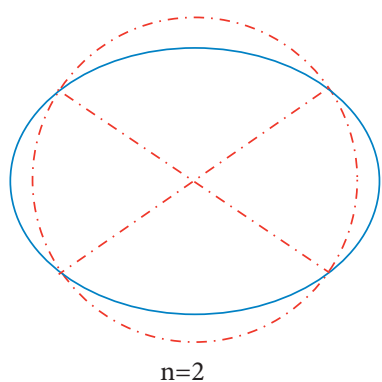

(b)
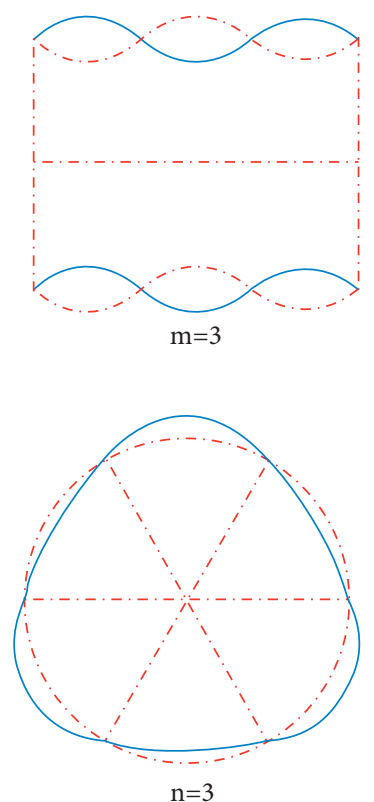

Figure 4: Vibration forms of the CTWW. (a) Axial vibration forms. (b) Circumferential vibration forms.

$$
\left\{\begin{array}{l}
u(z, \theta, t)=\sum_{m=0}^{\infty} \sum_{n=0}^{\infty} A_{m n} \phi_{m}(z) \sin (n \theta) q(t), \\
v(z, \theta, t)=\sum_{m=0}^{\infty} \sum_{n=0}^{\infty} B_{m n} \phi_{m}(z) \cos (n \theta) q(t), \\
w(z, \theta, t)=\sum_{m=0}^{\infty} \sum_{n=0}^{\infty} C_{m n} \frac{\mathrm{d} \phi_{m}(z)}{\mathrm{d}(z / L)} \cos (n \theta) q(t),
\end{array}\right.
$$

where $A_{m n}, B_{m n}$, and $C_{m n}$ are the $(m, n)$-th modal amplitudes in the tangential, radial, and axial directions, respectively, and $\phi_{m}(z)$ is the axial mode shape function that is defined as a beam mode shape function corresponding to the boundary conditions at both ends, which can be expressed as [43]

$$
\begin{aligned}
\phi_{m}(z)= & a_{1} \cosh \left(\frac{\lambda_{m} z}{L}\right)+a_{2} \cos \left(\frac{\lambda_{m} z}{L}\right) \\
& -\sigma_{m}\left[a_{3} \sin h\left(\frac{\lambda_{m} z}{L}\right)+a_{4} \sin \left(\frac{\lambda_{m} z}{L}\right)\right],
\end{aligned}
$$

where the values of $a_{i_{z}}\left(i_{z}=1,2,3,4\right), \lambda_{m}$, and $\sigma_{m}$ can be determined according to the boundary conditions of the beam. In this paper, the clamped-free boundary condition is considered, and the parameters of the beam function satisfy the following relationships:

$$
\left\{\begin{array}{l}
\cosh \lambda_{m} \cos \lambda_{m}=-1 \\
\sigma_{m}=\frac{\sinh \lambda_{m}-\sin \lambda_{m}}{\cosh \lambda_{m}+\cos \lambda_{m}} \\
a_{1}=a_{3}=1 \\
a_{2}=a_{4}=-1
\end{array}\right.
$$

By substituting equation (5) into (3) and performing Galerkin discretization, a set of ordinary differential equations can be obtained as follows:

$$
\left\{\begin{array}{l}
\int_{0}^{L} \int_{0}^{2 \pi}\left(L_{11} u+L_{12} v+L_{13} w\right) \phi_{m}(z) \sin (n \theta) R \mathrm{~d} \theta \mathrm{d} z=0, \\
\int_{0}^{L} \int_{0}^{2 \pi}\left(L_{21} u+L_{22} v+L_{23} w\right) \phi_{m}(z) \cos (n \theta) R \mathrm{~d} \theta \mathrm{d} z=0, \\
\int_{0}^{L} \int_{0}^{2 \pi}\left(L_{31} u+L_{32} v+L_{33} w\right) \frac{\mathrm{d} \phi_{m}(z)}{\mathrm{d}(z / L)} \cos (n \theta) R \mathrm{~d} \theta \mathrm{d} z=0 .
\end{array}\right.
$$

Further integrating equation (8), the frequency characteristic equation of the workpiece can be obtained as follows: 


$$
\left[\begin{array}{lll}
c_{11} & c_{12} & c_{13} \\
c_{21} & c_{22} & c_{23} \\
c_{31} & c_{32} & c_{33}
\end{array}\right]\left\{\begin{array}{l}
A_{m n} \\
B_{m n} \\
C_{m n}
\end{array}\right\}=0
$$

where $c_{i_{c} j_{c}}\left(i_{c}, j_{c}=1,2,3\right)$ are the undetermined coefficients as given in Appendix $B$, which are related to the natural frequency, geometric parameters, and material parameters of the workpiece.

Since equation (9) has nontrivial solutions, the determinant of the coefficient matrix must be zero, i.e.,

$$
\operatorname{det}\left(\left[c_{i_{c} j_{c}}\right]\right)=0, \quad\left(i_{c}, j_{c}=1,2,3\right)
$$

A characteristic equation of the workpiece can be obtained from equation (10) as

$$
\left(\omega_{m n}^{2}\right)^{3}+\gamma_{1}\left(\omega_{m n}^{2}\right)^{2}+\gamma_{2} \omega_{m n}^{2}+\gamma_{3}=0
$$

where $\omega_{m n}$ is the natural frequency corresponding to the $(m, n)$-th mode and $\gamma_{i_{r}}\left(i_{r}=1,2,3\right)$ are the coefficients which can be written as

$$
\left\{\begin{array}{l}
\gamma_{1}=-c_{13}-c_{21}-c_{32} \\
\gamma_{2}=c_{13} c_{21}+c_{13} c_{32}+c_{21} c_{32}-c_{11} c_{23}-c_{12} c_{33}-c_{22} c_{31} \\
\gamma_{3}=c_{13} c_{22} c_{31}+c_{11} c_{23} c_{32}+c_{12} c_{21} c_{33}-c_{11} c_{22} c_{33}-c_{12} c_{23} c_{33}-c_{13} c_{21} c_{32}
\end{array}\right.
$$

Based on equation (11), three natural frequency solutions are obtained and substituted into equation (9). As a result, ratios of modal amplitudes can be expressed as

$$
\left\{\begin{array}{l}
\frac{A_{m n}}{B_{m n}}=\frac{c_{22}\left(\omega_{m n}^{2}-c_{13}\right)+c_{12} c_{23}}{c_{11} c_{23}-\left(\omega_{m n}^{2}-c_{13}\right)\left(\omega_{m n}^{2}-c_{21}\right)}, \\
\frac{B_{m n}}{C_{m n}}=\frac{\left(\omega_{m n}^{2}-c_{13}\right)\left(\omega_{m n}^{2}-c_{21}\right)-c_{11} c_{23}}{c_{12}\left(\omega_{m n}^{2}-c_{21}\right)+c_{22} c_{11}} .
\end{array}\right.
$$

The above formulae reveal that there are three natural frequencies with regard to each combination $(m, n)$, and each frequency is related to one vibration direction. Among them, the lowest frequency value corresponds to the most flexural vibration direction, and the other two correspond to the in-plane vibrations. In this way, the calculated natural frequencies and mode shape functions of the CTWW will be used to establish the motion equation for the workpiecefixture system in next section.

\subsection{Motion Equation of the CTWW with Fixture Support} Constraints. For the workpiece-fixture system, an analytical mechanics method will be introduced to establish the motion equation. The generalized coordinates are used to determine the position of the system. The scalar functions such as kinetic energy, elastic strain energy, potential energy, dissipated energy, and the work done by external forces are used to describe the amount of motion. The Lagrange equation is used to obtain the relationships between multiple energies. And the specific modeling process will be described in detail as follows.

According to the research in [44], the displacement expression of the workpiece without support is still applicable for cases with support. Therefore, based on equation
(5), the kinetic energy Tof the CTWW under external forces can be expressed as

$$
\begin{aligned}
T= & \frac{\rho H}{2} \int_{0}^{L} \int_{0}^{2 \pi}\left(\left(\frac{\partial u(z, \theta, t)}{\partial t}\right)^{2}+\left(\frac{\partial v(z, \theta, t)}{\partial t}\right)^{2}\right. \\
& \left.+\left(\frac{\partial w(z, \theta, t)}{\partial t}\right)^{2}\right) R \mathrm{~d} \theta \mathrm{d} x=\frac{1}{2} m_{p} \dot{\mathbf{q}} \mathbf{M} \dot{\mathbf{q}}^{T}
\end{aligned}
$$

where $m_{p}$ represents the mass per unit area of the cylindrical shell and $\dot{\mathbf{q}}$ is a $1 \times(3 M \cdot N)$ matrix, and it can be expressed as $\dot{\mathbf{q}} \in R^{1 \times 3 M N}$, where $M$ and $N$ represent the number of related subitems corresponding to $m$ and $n$ in equation (5), respectively. Each of the generalized coordinate velocity matrix element is $\dot{q}=\partial q / \partial t$. The mass matrix $\mathbf{M}$ can be expressed as

$$
\mathbf{M}=\left[\begin{array}{cccc}
\mathbf{M}_{1} & & & 0 \\
& \mathbf{M}_{2} & & \\
& & \ddots & \\
& & & \mathbf{M}_{M N}
\end{array}\right]_{3 M N \times 3 M N}
$$

where the submatrix $\mathbf{M}_{i_{m}}\left(i_{m}=1,2, \ldots, M N\right)$ stands for the mass elements in the three directions corresponding to the $i_{m}$-th degree of freedom, each submatrix is a $3 \times 3$ matrix, and each element in the submatrix is given in Appendix C. These elements are related to the mode shape functions of the CTWW. The symbol "sym" in the matrix $\mathbf{M}$ is short for symmetry.

Deformation generates internal strain and stress and therefore creates elastic strain energy. Based on the classical thin shell theory, the elastic strain energy $U$ of the workpiece can be obtained as [45] 


$$
\begin{aligned}
U & =\int_{0}^{L} \int_{0}^{2 \pi} \frac{E H}{2\left(1-\mu^{2}\right)}\left\{\begin{array}{c}
{\left[\frac{\partial w}{\partial z}+\frac{1}{R}\left(\frac{\partial u}{\partial \theta}+v\right)\right]^{2}-\frac{2(1-\mu)}{R}\left[\frac{\partial w}{\partial z}\left(\frac{\partial u}{\partial \theta}+v\right)\right]+\frac{1-\mu}{2}\left(\frac{1}{R} \frac{\partial w}{\partial \theta}+\frac{\partial u}{\partial z}\right)^{2}} \\
+\frac{H^{2}}{12}\left[\frac{\partial^{2} v}{\partial z^{2}}+\frac{1}{R^{2}}\left(\frac{\partial^{2} v}{\partial \theta^{2}}-\frac{\partial u}{\partial \theta}\right)\right]^{2}-\frac{H^{2}(1-\mu)}{6 R^{2}}\left[\frac{\partial^{2} v}{\partial z^{2}}\left(\frac{\partial^{2} v}{\partial \theta^{2}}-\frac{\partial u}{\partial \theta}\right)\right]+\frac{H^{2}(1-\mu)}{6 R^{2}}\left(\frac{\partial^{2} v}{\partial z \partial \theta}-\frac{\partial u}{2 \partial z}\right)^{2}
\end{array}\right\} R \mathrm{~d} \theta \mathrm{d} z \\
& =\frac{1}{2} D \mathbf{q} \mathbf{K}_{w} \mathbf{q}^{T},
\end{aligned}
$$

where $\mathbf{K}_{w}$ is the stiffness matrix, which has the same dimension with the mass matrix $\mathbf{M}$, and the element in each submatrix is given in Appendix D.

Each fixture support can be modeled as a spring and a damping element, as shown in Figure 5. Under the excitation of external forces, the fixture support outputs normal force and tangential forces on the workpiece within the support area.

Assuming that the fixture support $a$ is located at $\left(z_{a}, \theta_{a}\right)$, the potential energy $P$ of all fixture supports can be written as

$$
\begin{aligned}
P & =\frac{1}{2} \sum_{a=1}^{p_{a}}\left[k_{\theta a} u^{2}\left(z_{a}, \theta_{a}\right)+k_{r a} v^{2}\left(z_{a}, \theta_{a}\right)+k_{z a} w^{2}\left(z_{a}, \theta_{a}\right)\right] \\
& =\frac{1}{2} \sum_{a=1}^{p_{a}} \mathbf{q} \mathbf{K}_{s} \mathbf{q}^{T},
\end{aligned}
$$

where $p_{a}$ is the number of the fixture supports, $k_{r a}, k_{z a}$, and $k_{\theta a}$ are the contact stiffness in the radial, axial, and tangential directions, respectively, and $\mathbf{K}_{s}$ is the stiffness matrix related to the fixture support, which also has the same expression as the mass matrix $\mathbf{M}$, and the submatrix in $\mathbf{K}_{s}$ can be written as

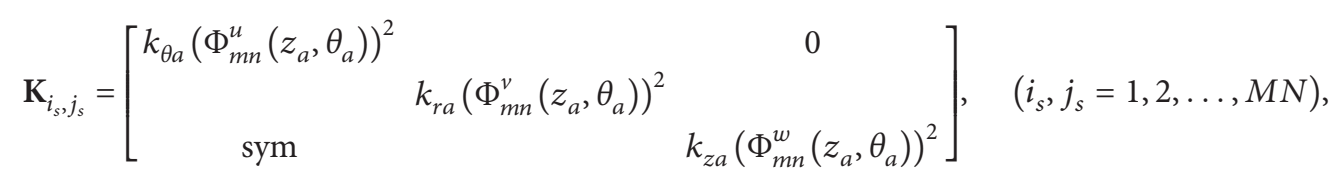

where $\Phi_{m n}^{u}\left(z_{a}, \theta_{a}\right), \Phi_{m n}^{v}\left(z_{a}, \theta_{a}\right)$, and $\Phi_{m n}^{w}\left(z_{a}, \theta_{a}\right)$ are the mode shape functions in three directions, which are functions of the support positions and can be obtained from Appendix C.

Since the workpiece-fixture system is an energy dissipative system, the damping effect cannot be ignored. To describe the energy dissipation of the system, it is necessary to comprehensively consider the damping characteristics of the workpiece and fixture supports. In the modeling process, the nonconservative damping forces are assumed to be viscous damping forces, and the Rayleigh dissipation function is used to express the dissipated energy $R_{d}$ of the system, which can be given as

$$
\begin{aligned}
R_{d}= & \frac{c_{w}}{2} \int_{0}^{L} \int_{0}^{2 \pi}\left(\left(\frac{\partial u(z, \theta, t)}{\partial t}\right)^{2}+\left(\frac{\partial v(z, \theta, t)}{\partial t}\right)^{2}+\left(\frac{\partial w(z, \theta, t)}{\partial t}\right)^{2}\right) R \mathrm{~d} \theta \mathrm{d} x \\
& +\frac{1}{2} \sum_{a=1}^{p_{a}} c_{s}\left(\left(\frac{\partial u(z, \theta, t)}{\partial t}\right)^{2}+\left(\frac{\partial v(z, \theta, t)}{\partial t}\right)^{2}+\left(\frac{\partial w(z, \theta, t)}{\partial t}\right)^{2}\right) \\
= & \frac{c_{w}}{2} \dot{\mathbf{q}} \mathbf{C}_{w} \dot{\mathbf{q}}^{T}+\frac{c_{s}}{2} \sum_{a=1}^{p_{a}} \dot{\mathbf{q}} \mathbf{C}_{s} \dot{\mathbf{q}}^{T},
\end{aligned}
$$




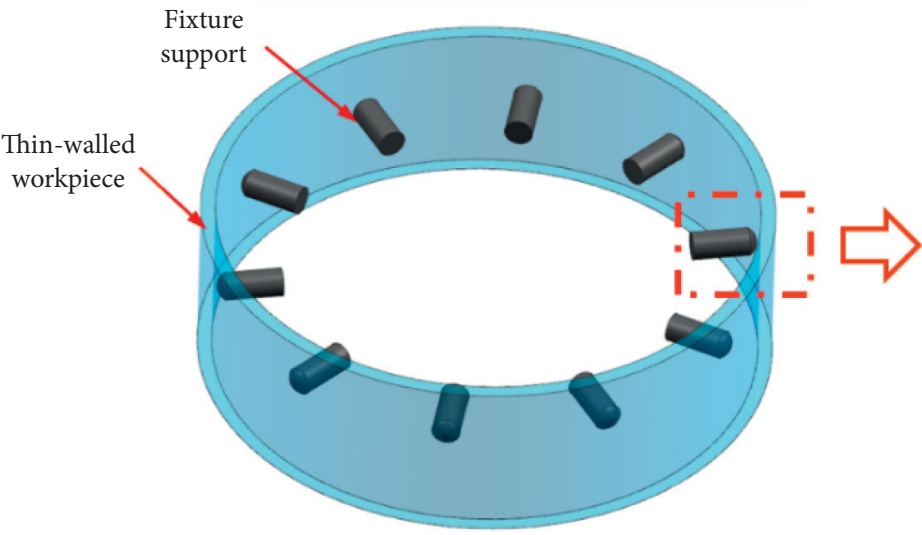

(a)

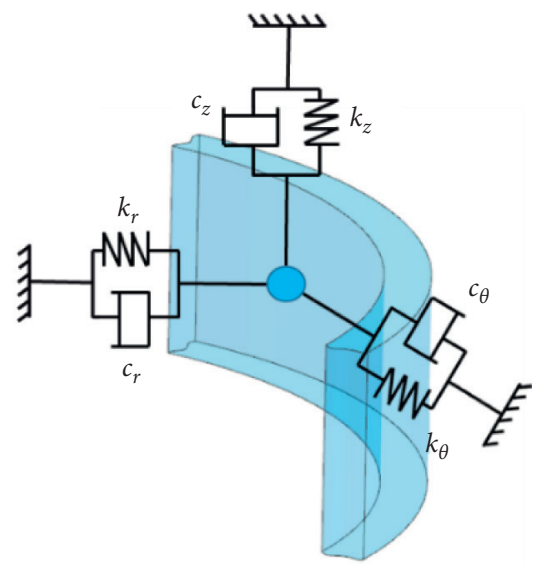

(b)

FIgURE 5: Illustration of the workpiece-fixture system. (a) CTWW with fixture support constraints. (b) Equivalent model of the fixture support.

where $c_{w}$ and $c_{s}$ are the damping coefficients of the workpiece and fixture support, respectively, and $\mathbf{C}_{w}$ and $\mathbf{C}_{s}$ are the damping matrices corresponding to the workpiece and fixture support, respectively.

Supposing that the workpiece is subjected to an external force $F_{e}$, the work $W$ done by $F_{e}$ can be obtained as

$$
W=F_{e, u} u+F_{e, v} v+F_{e, w} w=\mathbf{F}_{e} \boldsymbol{\Phi q}^{T},
$$

where $F_{e, u}, F_{e, v}$, and $F_{e, w}$ are the tangential, radial, and axial force components acting on the CTWW, respectively, and $\mathbf{F}_{e} \in R^{1 \times 3 M N}$ and $\Phi \in R^{3 M N \times 3 M N}$ are the external force matrix and mode shape matrix, respectively, which can be written as

$$
\left\{\begin{array}{l}
\mathbf{F}_{e}=\left[\begin{array}{llll}
\mathbf{F}_{e, 1} & \mathbf{F}_{e, 2} & \cdots & \mathbf{F}_{e, M N}
\end{array}\right]_{1 \times 3 M N}, \\
\boldsymbol{\Phi}=\left[\begin{array}{cccc}
\boldsymbol{\Phi}_{1} & & & 0 \\
& \boldsymbol{\Phi}_{2} & & \\
& & \ddots & \\
& & & \\
\text { sym } & & & \boldsymbol{\Phi}_{M N}
\end{array}\right]_{3 M N \times 3 M N}
\end{array} .\right.
$$

The submatrices in the above matrices can be expressed as

$$
\begin{cases}\mathbf{F}_{e, i_{e}}=\left[\begin{array}{lll}
F_{e, i_{e}, u} & F_{e, i_{e}, v} & F_{e, i_{e}, w}
\end{array}\right], & \left(i_{e}=1,2, \ldots, M N\right), \\
\boldsymbol{\Phi}_{i_{m}}=\left[\begin{array}{cc}
\Phi_{i_{m}, m n}^{u}(z, \theta) & 0 \\
& \Phi_{i_{m}, m n}^{v}(z, \theta) \\
\operatorname{sym} & \Phi_{i_{m}, m n}^{w}(z, \theta)
\end{array}\right], & \left(i_{m}=1,2, \ldots, M N\right) .\end{cases}
$$

According to the above derivations, the scalar functions of the system such as kinetic energy, elastic strain energy, potential energy, dissipated energy, and work have been given, which are used to describe the amount of motion of the system, and their relationship can be obtained based on the Lagrange equation as [46]

$$
\frac{\mathrm{d}}{\mathrm{d} t}\left(\frac{\partial T}{\partial \dot{q}}\right)-\frac{\partial T}{\partial q}+\frac{\partial R_{d}}{\partial \dot{q}}+\frac{\partial(U+P)}{\partial q}=\frac{\partial W}{\partial q} .
$$

By substituting equations (14), (16), (17), (19), and (20) into (23), the motion equation of the workpiece-fixture system can be expressed in terms of generalized coordinates and mode shapes as follows:

$$
m_{p} \mathbf{M} \ddot{\mathbf{q}}+\left(c_{w} \mathbf{C}_{w}+c_{s} \mathbf{C}_{s}\right) \dot{\mathbf{q}}+\left(D \mathbf{K}_{w}+\sum_{a=1}^{p_{a}} \mathbf{K}_{s}\right) \mathbf{q}=\mathbf{F}_{e} \boldsymbol{\Phi} .
$$

Considering the complexity of the system damping, it is approximated by Rayleigh damping. The motion equation of the system can be written as

$$
\mathbf{M} \ddot{\mathbf{q}}+\mathbf{C} \dot{\mathbf{q}}+\mathbf{K q}=\mathbf{F},
$$


where

$$
\left\{\begin{array}{l}
\mathbf{C}=\alpha \mathbf{M}+\frac{\beta\left(D \mathbf{K}_{w}+\sum_{a=1}^{p_{a}} \mathbf{K}_{s}\right)}{m_{p}}, \\
\mathbf{K}=\frac{\left(D \mathbf{K}_{w}+\sum_{a=1}^{p_{a}} \mathbf{K}_{s}\right)}{m_{p}}, \\
\mathbf{F}=\frac{\mathbf{F}_{e} \mathbf{\Phi}}{m_{p}},
\end{array}\right.
$$

where $\alpha$ and $\beta$ are constants.

In this way, the motion equation of the workpiece-fixture system is established. It can be seen that the proposed method can considerably reduce the computational complexity of the vector relationship by using the scalar functions, thereby making the modeling of the complex system easier and facilitating the prediction of the FRFs for the workpiece-fixture system in next section.

\section{Frequency Response Function of the Workpiece-Fixture System}

Fixture support increases the rigidity of the CTWW and also changes its dynamic characteristics. In this section, the workpiece-fixture system is regarded as a linear time-invariant (LTI) system, and the FRF is predicted.

By taking Fourier transform on equation (25), it becomes

$$
\left(\mathbf{K}-\omega^{2} \mathbf{M}+j \omega \mathbf{C}\right) \mathbf{q}(\omega)=\mathbf{F}(\omega) .
$$

Based on equation (27), the motion equation of the undamped free vibration system is given by

$$
\left(\mathbf{K}-\omega^{2} \mathbf{M}\right) \mathbf{q}(\omega)=0 .
$$

From equation (28), the natural frequencies can be calculated for each set of $(m, n)$. Among the three frequencies in axial, radial, and tangential directions, the minimum value corresponds to the radial natural frequency, which will be analyzed in detail.

For the LTI system, the system response can be represented using the mode superposition method [47]. Thus, the response vector $\mathbf{q}$ can be written as

$$
\mathbf{q}(\omega)=\mathbf{\Psi} \mathbf{p}(\omega)
$$

where $\Psi=\left[\begin{array}{llll}\Psi_{1} & \Psi_{2} & \cdots & \Psi_{N_{d}}\end{array}\right]$ is a new mode shape matrix, $\Psi_{r}\left(r=1,2, \ldots, N_{d}\right)$ is the $r$-th order mode shape vector, $\mathbf{p}=\left[\begin{array}{llll}p_{1}(\omega) & p_{2}(\omega) & \cdots & p_{N_{d}}(\omega)\end{array}\right]^{T}$ is the modal coordinate vector, and $N_{d}$ is the number of modes.

Substituting equation (29) into (27) and premultiplying it by $\Psi^{T}$, the $r$-th order modal equation of the system can be obtained on the basis of the orthogonality of the mode shapes:

$$
\left(K_{r}-\omega^{2} M_{r}+j \omega C_{r}\right) p_{r}=F_{r}
$$

where $F_{r}$ is the $r$-th order modal force and $K_{r}, M_{r}$, and $C_{r}$ are the $r$-th order modal stiffness, modal mass, and modal damping, respectively.

According to the above equation, the $r$-th order modal coordinate can be obtained as

$$
p_{r}=\frac{F_{r}}{K_{r}-\omega^{2} M_{r}+j \omega C_{r}}
$$

where $F_{r}=\Psi_{r}^{T} \mathbf{F}(\omega)=\sum_{l=1}^{p_{n}} \varphi_{l r} f_{j_{r}}(\omega),\left(j_{r}=1,2, \ldots, p_{n}\right), \varphi_{l r}$ is the mode shape of the $r$-th order mode at the measurement point $l$, and $p_{n}$ is the number of measurement points.

In this section, single point excitation is considered. Supposing that the exciting force is applied to point $s$, the force vector can be written as

$$
\mathbf{F}(\omega)=\left[0 \cdots 0 \cdots f_{s}(\omega) \cdots 0\right]^{T}
$$

and the $r$-th order modal force becomes

$$
F_{r}=\varphi_{s r} f_{s}(\omega)
$$

Since the workpiece-fixture system is regarded as a LTI system, the response at any point $l$ can be expressed as

$$
q_{l}(\omega)=\varphi_{l 1} p_{1}(\omega)+\varphi_{l 2} p_{2}(\omega)+\cdots+\varphi_{l N} p_{N}(\omega)=\sum_{r=1}^{N_{d}} \varphi_{l r} p_{r}(\omega)
$$

By substituting equations (31) and (33) into (34), the frequency response function between the measurement point $l$ and the excitation point $s$ can be given by

$$
\frac{q_{l}(\omega)}{f_{s}(\omega)}=H_{l s}(\omega)=\sum_{r=1}^{N_{d}} \frac{\varphi_{l r} \varphi_{s r}}{K_{r}-\omega^{2} M_{r}+j \omega C_{r}},
$$

i.e.,

$$
H_{l s}(\omega)=\sum_{r=1}^{N_{d}} \frac{1}{K_{e r}\left[\left(1-\bar{\omega}_{r}^{2}+2 j \xi_{r} \bar{\omega}_{r}\right)\right]},
$$

where $K_{e r}=\left(K_{r} / \varphi_{l r} \varphi_{s r}\right)$ in unit $\mathrm{N} / \mathrm{m}, \bar{\omega}_{r}=\left(\omega / \omega_{r}\right)$, $\xi_{r}=\left(C_{r} / 2 M_{r} \omega_{r}\right)$, and $\omega_{r}$ is the $r$-th order natural frequency of the system in unit rad/s.

Through the above analysis, the FRFs of the workpiecefixture system are obtained, in which a complete mode set is used, that is, all modes are superimposed. However, in actual analysis, the modal truncation method is widely used to reduce the computational burden. The dynamic characteristics of the system are investigated by selecting the first several modes, which will be applied in subsequent verification of the proposed method.

\section{Verification}

To verify the feasibility of the proposed method, a series of numerical calculation and experimental modal tests are carried out on a specifically designed workpiece-fixture system. 


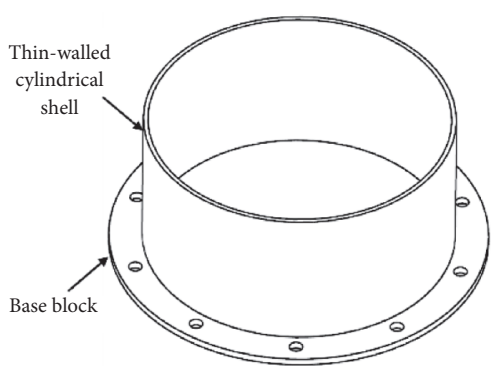

(a)

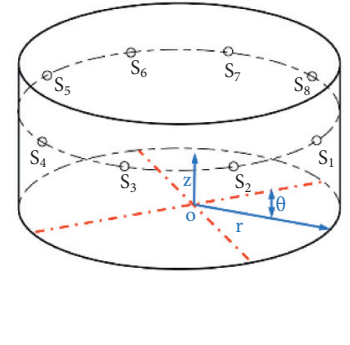

(b)

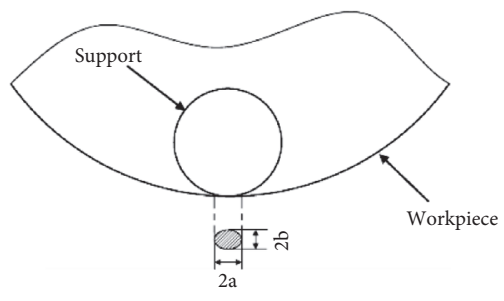

(c)

FIgure 6: The designed CTWW for verification. (a) Schematic illustration of CTWW. (b) Fixture support layout scheme. (c) Ball head support and workpiece.

4.1. Workpiece and Fixture Support. A specific CTWW and fixture support layout are designed to validate the proposed method, as shown in Figure 6. The workpiece in Figure 6(a) is composed of two parts: the base block and the thin-walled cylindrical shell. For the base block, its outer diameter is $250 \mathrm{~mm}$ and inner diameter is $200 \mathrm{~mm}$. To achieve the clamped boundary conditions at the bottom of the workpiece, the base block is designed to have ten cylindrical holes that are used to bolt the workpiece to the fixture base. For the thin-walled cylindrical shell, the outer diameter is $206 \mathrm{~mm}$, inner diameter is $200 \mathrm{~mm}$, and the height is $100 \mathrm{~mm}$. Furthermore, the material of the workpiece is aluminum alloy 6061, which has the following physical parameters [44], as listed in Table 1. Without loss of generality, a support layout strategy used in actual on-site processing is presented, as shown in Figure 6(b). The specific locations of the fixture supports are listed in Table 2.

In this section, the support is designed with a ball head, which can ensure full contact with the inner wall of the workpiece. Under the action of the supporting force, the contact area between the support and the workpiece is elliptical, as shown in Figure 6(c). Since the rigidity of the workpiece in the radial direction is the weakest, only the supporting effect in this direction is considered in the study. Thus, the contact stiffness between the support and the workpiece is

$$
k_{s}=\frac{\mathrm{d} F_{s}}{\mathrm{~d} \delta_{s}}
$$

where $F_{s}$ is the radial support force and $\delta_{s}$ is the elastic deformation, which can be obtained based on Hertz contact, and it is [48]

$$
\delta_{s}=\delta^{*}\left[\frac{3 F_{s}}{2 \sum \rho_{t}}\left(\frac{1-v_{f}^{2}}{E_{f}}+\frac{1-v_{w}^{2}}{E_{w}}\right)\right]^{2 / 3} \frac{\sum \rho_{t}}{2},
$$

where $\delta^{*}$ is a dimensionless parameter (the specific expression is given in Appendix E), $v_{f}$ and $v_{w}$ are Poisson's ratios of the support and the workpiece, respectively, $E_{f}$ and $E_{w}$ are the elastic moduli of the support and the workpiece, respectively, and $\sum \rho_{t}$ is the sum of curvature of the contact part of the workpiece and the support.
TABLe 1: Physical parameters of the aluminum alloy 6061 .

\begin{tabular}{lcc}
\hline Young's modulus $(\mathrm{Pa})$ & Poisson's ratio & Density $\left(\mathrm{kg} / \mathrm{m}^{3}\right)$ \\
\hline $68.9 \times 10^{9}$ & 0.33 & 2800 \\
\hline
\end{tabular}

Table 2: Fixture support layout as shown in Figure 6(b).

\begin{tabular}{lc}
\hline Supports & Positions $(z, \theta, r)$ \\
\hline$S 1$ & $(60,45,100)$ \\
S2 & $(60,90,100)$ \\
S3 & $(60,135,100)$ \\
S4 & $(60,180,100)$ \\
S5 & $(60,225,100)$ \\
S6 & $(60,270,100)$ \\
S7 & $(60,325,100)$ \\
S8 & $(60,360,100)$ \\
\hline
\end{tabular}

After the workpiece and fixture support layout are determined, a series of numerical calculation and hammer tests can be carried out to verify the proposed method.

4.2. Numerical Calculation and Hammer Tests. Since the low-order modes have a great influence on the workpiece, the first two modes are considered in this paper. According to equation (36), the FRFs of the workpiece-fixture system can be obtained. To investigate the effects of fixture support on workpiece dynamic characteristics, the point with weak local stiffness is a good choice for comparison. Here, points along the circumferential path at the axial coordinate of $95 \mathrm{~mm}$ are selected to calculate the FRFs, and the predicted results of the workpiece with and without fixture support are shown in Figure 7. In the calculation process, the damping coefficients of the workpiece-fixture system are decided by means of impact test, where the damping ratio can be obtained. In addition, for the case of support, the diameter of the support ball head is $7 \mathrm{~mm}$, and the support force is $15 \mathrm{~N}$. It can be seen that after the fixture support is added to the workpiece, the FRF has the phenomenon of natural frequency value shift and amplitude reduction.

To verify the accuracy of the predicted FRFs, hammer tests are performed, as shown in Figure 8. Among them, the size of the experimental workpiece is the same as that of the designed structure, as shown in Figure 6(a), and the specific fixture support layout is consistent with that in Figure 6(b). 


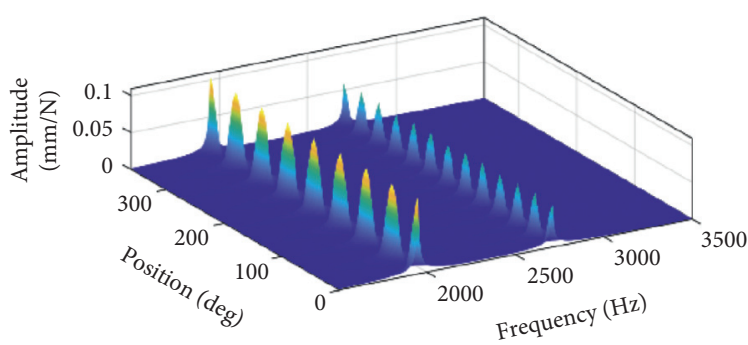

(a)

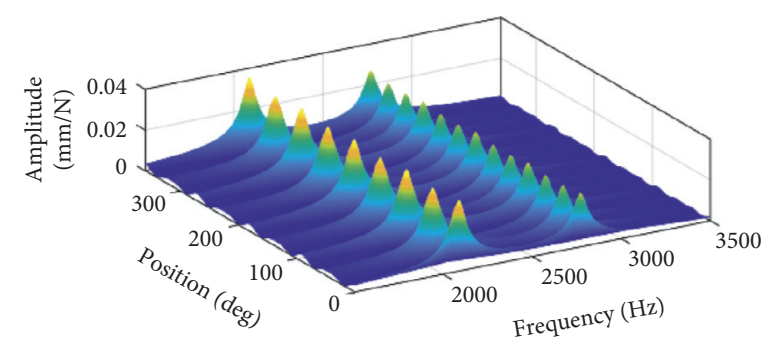

(b)

FIGURE 7: Comparisons of the predicted FRFs of workpiece. (a) Without support. (b) With support.

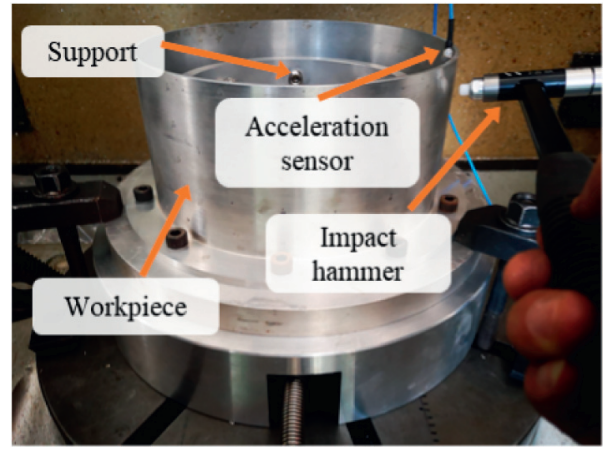

(a)

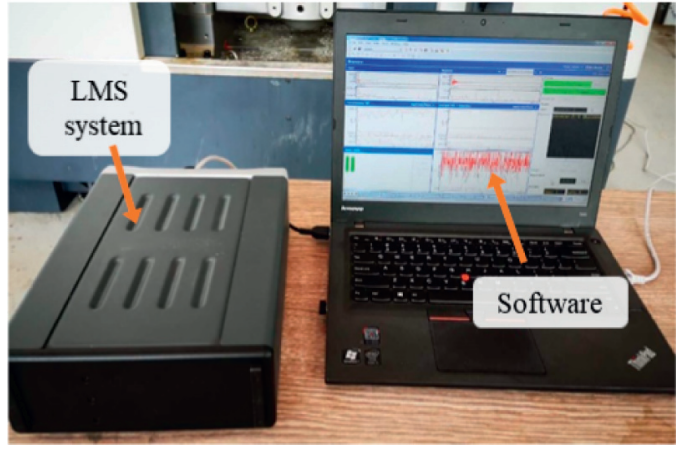

(b)

FIGURE 8: Experimental setup for hammer tests.

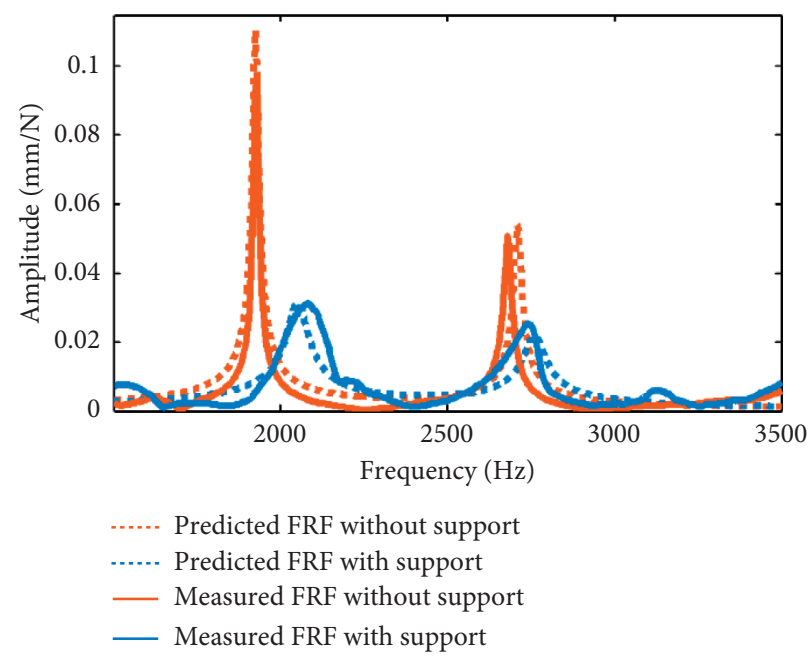

(a)

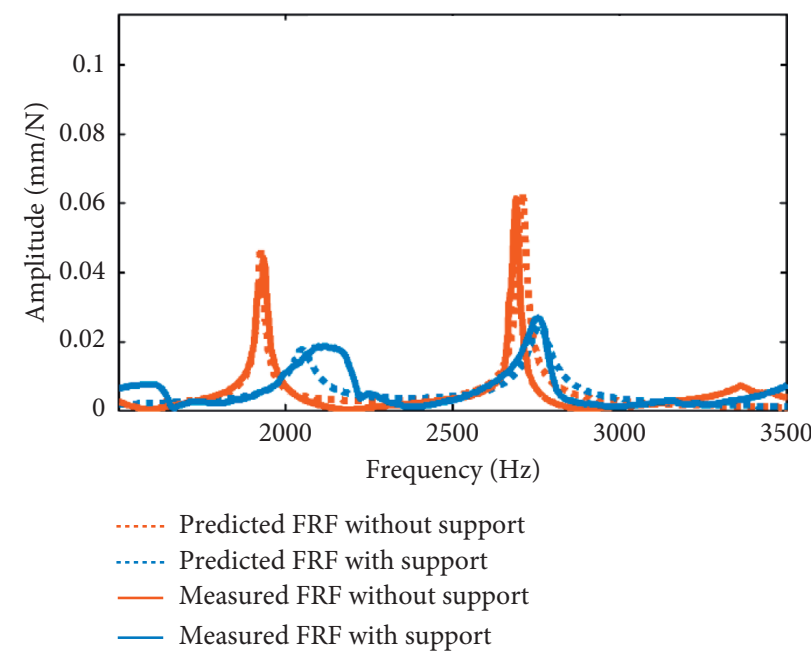

(b)

Figure 9: Comparisons of the FRFs of the workpiece with and without fixture support. (a) Point 1. (b) Point 2.

Moreover, the diameter of the support ball head and the support force are also in accord with those in the prediction. During the test, an impact hammer PCB 086C01 is selected as the excitation source and an acceleration sensor $\mathrm{PCB}$ $356 \mathrm{~A} 01$ is used to obtain the vibration response of the workpiece. The transfer function between the excitation point and the response point can be obtained by the ratio of acceleration to force, and then it can be integrated to obtain the ratio of displacement to force in the LMS data acquisition system SCADAS SCM202.

With the help of the above experimental setup, the FRFs of the workpiece with and without fixture support can be obtained. In order to analyze the effects of fixture support on the dynamic characteristics of the workpiece, two points P1 $(95,3,103)$ and P2 $(95,33,103)$ on the workpiece are selected, for which the measured FRFs are shown in Figure 9. 
TABLE 3: Comparison of the predicted and measured natural frequency values.

\begin{tabular}{lccc}
\hline Support layout & Modes & Predicted $(\mathrm{Hz})$ & Measured $(\mathrm{Hz})$ \\
\hline \multirow{2}{*}{ Without support } & 1 & 1926 & 1930 \\
& 2 & 2710 & 2685 \\
\hline \multirow{2}{*}{ With support } & 1 & 2052 & 2081 \\
& 2 & 2761 & 2743 \\
\hline
\end{tabular}

At the same time, the predicted FRFs of the two points are also included for comparison. It can be observed that the measured results are basically consistent with the predicted results.

In order to further observe the changes in the dynamic characteristics of the workpiece after adding support, the natural frequency values and amplitudes of the FRFs are compared and analyzed, respectively. The natural frequency values of the workpiece with and without fixture support are listed in Table 3. It can be seen that the predicted natural frequency values agree well with the experimental values, and the errors are within 5\%. Moreover, it can be noted that the support has a greater influence on the natural frequency value of the low-order mode of the workpiece. Specifically, the frequency change of the first-order mode is much larger than that of the second-order mode.

As shown in Figure 9, the amplitudes are effectively reduced after adding fixture supports. The amplitude of each mode also changes with the measured position, which is attributed to the influence of mode shape of the workpiece. Experimental modal tests reveal that the amplitude changes of the two points are in line with the predicted results. The slight discrepancies between the predicted results and the test results may be caused by the measurement error in the test process or the simplification error of the complex interaction between workpiece and fixture support, which deserves further exploration in the future.

\section{Conclusions}

In this paper, a comprehensive analytical solution is proposed to investigate effects of fixture supports on the dynamic characteristics of CTWWs. During the modeling process, a number of methods are used, such as the vector mechanics method, the analytical mechanics method, the variable separation method, the modal superposition method, and the modal truncation method. These methods can simplify the analysis of the complex multi-degree-offreedom system and avoid tedious mathematical calculation. According to the developed model, effects of fixture support on workpiece dynamic characteristics can be analyzed effectively. The feasibility of the proposed method is verified on a specifically designed workpiece-fixture system by means of numerical calculation and experimental hammer tests. The FRFs of different points on the workpiece with and without support are compared and analyzed, and some important conclusions are drawn as follows:

(1) The FRFs of the CTWW with fixture support constraints can be accurately predicted by using the proposed method, which are in good agreement with the experimental results.

(2) The dynamics of the CTWW can be improved by adding fixture support, where the FRFs of the system have changed a lot. Specifically, the natural frequency value increases and the amplitude decreases, especially for low-order modes.

(3) The influence of the fixture support on the dynamics of the CTWW can be explained clearly from the mechanism, that is, the stiffness of the support can affect the natural frequency value of the FRF, and the damping of the support can affect the amplitude of the FRF.

(4) In the design of fixtures for thin-walled workpieces, adding auxiliary supports is an effective method, which can increase the rigidity of the system and reduce the vibration of the system.

The main work of this paper is to investigate effects of fixture support on the dynamic characteristics of CTWW. Further, there are some other problems that need to be considered in the future, such as the effects of different support types and number of supports on the dynamic characteristics of thin-walled workpiece. In addition, the complex interaction between the support and the workpiece is an important factor that affects the accuracy of the prediction, which also needs to be explored in the future.

\section{Appendix}

\section{A. Differential Operators in Equation (3)}

The differential operators of thin-walled workpiece in equation (3) are

$$
\begin{aligned}
& L_{11}=K \frac{1+\mu}{2 R} \frac{\partial^{2}}{\partial \theta \partial z}, \\
& L_{12}=K \frac{\mu}{R} \frac{\partial}{\partial z} \\
& L_{13}=K \frac{\partial^{2}}{\partial z^{2}}+K \frac{1-\mu}{2 R^{2}} \frac{\partial^{2}}{\partial \theta^{2}}, \\
& L_{21}=K \frac{1-\mu}{2} \frac{\partial^{2}}{\partial z^{2}}+K \frac{1}{R^{2}} \frac{\partial^{2}}{\partial \theta^{2}}+D \frac{1-\mu}{2 R^{2}} \frac{\partial^{2}}{\partial z^{2}}+D \frac{1}{R^{4}} \frac{\partial^{2}}{\partial \theta^{2}}, \\
& L_{22}=K \frac{1}{R^{2}} \frac{\partial}{\partial \theta}-D \frac{1}{R^{4}} \frac{\partial^{3}}{\partial \theta^{3}}-D \frac{1}{R^{2}} \frac{\partial^{3}}{\partial z^{2} \partial \theta}, \\
& L_{23}=K \frac{1+\mu}{2 R} \frac{\partial^{2}}{\partial \theta \partial z}, \\
& L_{31}=K \frac{1}{R^{2}} \frac{\partial}{\partial \theta}-D \frac{1}{R^{4}} \frac{\partial^{3}}{\partial \theta^{3}}-D \frac{1}{R^{2}} \frac{\partial^{3}}{\partial z^{2} \partial \theta}, \\
& L_{32}=K \frac{1}{R^{2}}+D \frac{\partial^{4}}{\partial z^{4}}+D \frac{1}{R^{4}} \frac{\partial^{4}}{\partial \theta^{4}}+D \frac{2}{R^{2}} \frac{\partial^{4}}{\partial z^{2} \partial \theta^{2}}, \\
& L_{33}=K \frac{\mu}{R} \frac{\partial}{\partial z} .
\end{aligned}
$$




\section{B. Undetermined Coefficients in Equation (9)}

The expressions of the undetermined coefficients $c_{i_{c} j_{c}}$ in equation (9) are given as follows:

$$
\begin{aligned}
& c_{11}=K \frac{n(1+\mu)}{2 \rho H R} \frac{\int_{0}^{L} \phi_{m}^{\prime}(z) \phi_{m}^{\prime \prime \prime}(z) \mathrm{d} z}{\int_{0}^{L} \phi_{m}^{\prime}(z) \phi_{m}^{\prime}(z) \mathrm{d} z}, \\
& c_{12}=K \frac{\mu}{\rho H R} \frac{\int_{0}^{L} \phi_{m}^{\prime}(z) \phi_{m}^{\prime \prime \prime}(z) \mathrm{d} z}{\int_{0}^{L} \phi_{m}^{\prime}(z) \phi_{m}^{\prime}(z) \mathrm{d} z}, \\
& c_{13}=\omega_{m n}^{2}+\frac{K}{\rho H}\left[\frac{\int_{0}^{L} \phi_{m}(z) \phi_{m}^{\prime \prime}(z) \mathrm{d} z}{\int_{0}^{L} \phi_{m}(z) \phi_{m}(z) \mathrm{d} z}-\frac{n^{2}(1-\mu)}{2 R^{2}}\right] \text {, } \\
& c_{21}=\omega_{m n}^{2}+\frac{\left(K R^{2}+D\right)}{\rho H R^{2}}\left(\frac{1-\mu}{2} \frac{\int_{0}^{L} \phi_{m}(z) \phi_{m}^{\prime \prime}(z) \mathrm{d} z}{\int_{0}^{L} \phi_{m}(z) \phi_{m}(z) \mathrm{d} z}-\frac{n^{2}}{R^{2}}\right), \\
& c_{22}=-\frac{D n}{\rho H R^{2}}\left(\frac{\int_{0}^{L} \phi_{m}(z) \phi_{m}^{\prime \prime}(z) \mathrm{d} z}{\int_{0}^{L} \phi_{m}(z) \phi_{m}(z) \mathrm{d} z}-\frac{n^{2}}{R^{2}}\right)+\frac{K n}{\rho H R^{2}}, \\
& c_{23}=-K \frac{n(1+\mu)}{2 \rho H R} \frac{\int_{0}^{L} \phi_{m}^{\prime}(z) \phi_{m}^{\prime \prime \prime}(z) \mathrm{d} z}{\int_{0}^{L} \phi_{m}^{\prime}(z) \phi_{m}^{\prime}(z) \mathrm{d} z}, \\
& c_{31}=\frac{D n}{\rho H R^{2}}\left(\frac{\int_{0}^{L} \phi_{m}(z) \phi_{m}^{\prime \prime}(z) \mathrm{d} z}{\int_{0}^{L} \phi_{m}(z) \phi_{m}(z) \mathrm{d} z}-\frac{n^{2}}{R^{2}}\right)-\frac{K n}{\rho H R^{2}}, \\
& c_{32}=\omega_{m n}^{2}+D \frac{n^{2}}{\rho H R^{2}}\left(2 \frac{\int_{0}^{L} \phi_{m}(z) \phi_{m}^{\prime \prime}(z) \mathrm{d} z}{\int_{0}^{L} \phi_{m}(z) \phi_{m}(z) \mathrm{d} z}-\frac{n^{2}}{R^{2}}\right)+\frac{K}{\rho H R^{2}}+\frac{D}{\rho H} \frac{\int_{0}^{L} \phi_{m}(z) \phi_{m}^{\prime \prime \prime \prime}(z) \mathrm{d} z}{\int_{0}^{L} \phi_{m}(z) \phi_{m}(z) \mathrm{d} z}, \\
& c_{33}=-K \frac{\mu}{\rho H R} \frac{\int_{0}^{L} \phi_{m}^{\prime}(z) \phi_{m}^{\prime \prime}(z) \mathrm{d} z}{\int_{0}^{L} \phi_{m}^{\prime}(z) \phi_{m}^{\prime}(z) \mathrm{d} z}
\end{aligned}
$$

where the superscripts of $\phi_{m}^{\prime}(z), \phi_{m}^{\prime \prime}(z), \phi_{m}^{\prime \prime \prime}(z)$, and $\phi_{m}^{\prime \prime \prime \prime}(z)$ represent the corresponding derivative order.

\section{Element Expression in Equation (15)}

The expression of each element in the submatrix in equation (15) is

$$
\begin{aligned}
& M_{11}=\rho H \int_{0}^{L} \int_{0}^{2 \pi} \Phi_{m n}^{u}(z, \theta) \Phi_{m n}^{u}(z, \theta) R \mathrm{~d} \theta \mathrm{d} z, \\
& M_{22}=\rho H \int_{0}^{L} \int_{0}^{2 \pi} \Phi_{m n}^{v}(z, \theta) \Phi_{m n}^{v}(z, \theta) R \mathrm{~d} \theta \mathrm{d} z, \\
& M_{33}=\rho H \int_{0}^{L} \int_{0}^{2 \pi} \Phi_{m n}^{w}(z, \theta) \Phi_{m n}^{w}(z, \theta) R \mathrm{~d} \theta \mathrm{d} z, \\
& M_{12}=M_{13}=M_{21}=M_{23}=M_{31}=M_{32}=0,
\end{aligned}
$$

where $\Phi_{m n}^{u}(z, \theta), \Phi_{m n}^{v}(z, \theta)$, and $\Phi_{m n}^{w}(z, \theta)$ are the mode shape functions in three directions, and they can be expressed as

$$
\begin{aligned}
& \Phi_{m n}^{u}(z, \theta)=A_{m n} \phi_{m}(z) \sin (n \theta), \\
& \Phi_{m n}^{v}(z, \theta)=B_{m n} \phi_{m}(z) \cos (n \theta), \\
& \Phi_{m n}^{w}(z, \theta)=C_{m n} \frac{\mathrm{d} \phi_{m}(z)}{\mathrm{d}(z / L)} \cos (n \theta) .
\end{aligned}
$$

\section{Element Expression in Equation (16)}

The expression of each element in the submatrix in equation (16) is 


$$
\begin{aligned}
& K_{11}=\frac{E H}{1-\mu^{2}} \int_{0}^{L} \int_{0}^{2 \pi}\left[\left(\frac{\partial \Phi_{m n}^{w}(z, \theta)}{\partial z}\right)^{2}+\frac{1-\mu}{2 R^{2}}\left(\frac{\partial \Phi_{m n}^{w}(z, \theta)}{\partial \theta}\right)^{2}\right] R \mathrm{~d} \theta \mathrm{d} z \\
& K_{12}=K_{21}=\frac{E H}{2\left(1-\mu^{2}\right)} \int_{0}^{L} \int_{0}^{2 \pi}\left(\frac{2 \mu}{R} \frac{\partial \Phi_{m n}^{w}(z, \theta)}{\partial z} \frac{\partial \Phi_{m n}^{u}(z, \theta)}{\partial \theta}+\frac{1-\mu}{R} \frac{\partial \Phi_{m n}^{w}(z, \theta)}{\partial \theta} \frac{\partial \Phi_{m n}^{u}(z, \theta)}{\partial z}\right) R \mathrm{~d} \theta \mathrm{d} z \\
& K_{13}=K_{31}=\frac{E H}{2\left(1-\mu^{2}\right)} \int_{0}^{L} \int_{0}^{2 \pi}\left(\frac{2 \mu}{R} \frac{\partial \Phi_{m n}^{w}(z, \theta)}{\partial z} \Phi_{m n}^{v}(z, \theta)\right) R \mathrm{~d} \theta \mathrm{d} z, \\
& K_{22}=\frac{E H}{1-\mu^{2}} \int_{0}^{L} \int_{0}^{2 \pi}\left[\left(\frac{1}{R^{2}}+\frac{H^{2}}{12 R^{4}}\right)\left(\frac{\partial \Phi_{m n}^{u}(z, \theta)}{\partial \theta}\right)^{2}+\left(\frac{H^{2}}{24 R^{2}}+\frac{1}{2}\right)(1-\mu)\left(\frac{\partial \Phi_{m n}^{u}(z, \theta)}{\partial z}\right)^{2}\right] R \mathrm{~d} \theta \mathrm{d} z \\
& K_{23}=K_{32}=\frac{E H}{2\left(1-\mu^{2}\right)} \int_{0}^{L} \int_{0}^{2 \pi}\left[\begin{array}{c}
\frac{2}{R^{2}} \frac{\partial \Phi_{m n}^{u}(z, \theta)}{\partial \theta} \Phi_{m n}^{v}(z, \theta)-\frac{\mu H^{2}}{6 R^{2}} \frac{\partial \Phi_{m n}^{u}(z, \theta)}{\partial \theta} \frac{\partial^{2} \Phi_{m n}^{v}(z, \theta)}{\partial z^{2}} \\
-\frac{H^{2}}{6 R^{4}} \frac{\partial \Phi_{m n}^{u}(z, \theta)}{\partial \theta} \frac{\partial^{2} \Phi_{m n}^{v}(z, \theta)}{\partial \theta^{2}}-\frac{H^{2}(1-\mu)}{6 R^{2}} \frac{\partial \Phi_{m n}^{u}(z, \theta)}{\partial z} \frac{\partial^{2} \Phi_{m n}^{v}(z, \theta)}{\partial z \partial \theta}
\end{array}\right] R \mathrm{~d} \theta \mathrm{d} z \\
& K_{33}=\frac{E H}{1-\mu^{2}} \int_{0}^{L} \int_{0}^{2 \pi}\left[\begin{array}{c}
\left(\frac{\partial \Phi_{m n}^{v}(z, \theta)}{R}\right)^{2}+\frac{H^{2}}{12 R^{4}}\left(\frac{\partial^{2} \Phi_{m n}^{v}(z, \theta)}{\partial \theta^{2}}\right)^{2}+\frac{\mu H^{2}}{6 R^{2}} \frac{\partial^{2} \Phi_{m n}^{v}(z, \theta)}{\partial z^{2}} \frac{\partial^{2} \Phi_{m n}^{v}(z, \theta)}{\partial \theta^{2}} \\
+\frac{H^{2}}{12}\left(\frac{\partial^{2} \Phi_{m n}^{v}(z, \theta)}{\partial z^{2}}\right)^{2}+\frac{H^{2}(1-\mu)}{6 R^{2}}\left(\frac{\partial^{2} \Phi_{m n}^{v}(z, \theta)}{\partial z \partial \theta}\right)^{2}
\end{array}\right] R \mathrm{~d} \theta \mathrm{d} z
\end{aligned}
$$

\section{E. Dimensionless Parameter in Equation (38)}

The expression of dimensionless parameter $\delta^{*}$ in equation (38) is

$$
\delta^{*}=\frac{2 \Gamma}{\pi}\left(\frac{\pi}{2 k_{a b}^{2} \Sigma}\right)^{1 / 3}
$$

where

$$
\begin{aligned}
k_{a b} & =\frac{a}{b} \\
\Gamma & =\int_{0}^{2 \pi}\left[1-\left(1-\frac{1}{k_{a b}^{2}}\right) \sin \varphi^{2}\right]^{-(1 / 2)} \mathrm{d} \varphi, \\
\sum & =\int_{0}^{2 \pi}\left[1-\left(1-\frac{1}{k_{a b}^{2}}\right) \sin \varphi^{2}\right]^{(1 / 2)} \mathrm{d} \varphi
\end{aligned}
$$

where $k_{a b}$ is the ellipticity of the contact ellipse and $\Gamma$ and $\sum$ are the complete elliptic integrals of the first and second types, respectively, and they can be obtained according to the related formulae of [49].

\section{Data Availability}

The data used to support the findings of this study are included within the article and are available from the corresponding author upon request.

\section{Conflicts of Interest}

The authors declare that there are no conflicts of interest regarding the publication of this paper.

\section{Acknowledgments}

This study was supported by the NKRDPC (grant no. 2018YFA0704603) and the National Natural Science Foundation of China (grant no. 51905076).

\section{References}

[1] Y. Sun and S. Jiang, "Predictive modeling of chatter stability considering force-induced deformation effect in milling thinwalled parts," International Journal of Machine Tools and Manufacture, vol. 135, pp. 38-52, 2018.

[2] S. Jiang and Y. Sun, "Stability analysis for a milling system considering multi-point-contact cross-axis mode coupling and cutter run-out effects," The Mechanical Systems and Signal Processing, vol. 141, p. 106452, 2020.

[3] J. Niu, J. Xu, F. Ren, Y. Sun, and D. Guo, "A short review on milling dynamics in low-stiffness cutting conditions: modeling and analysis," Journal of Advanced Manufacturing Science and Technology, vol. 1, no. 1, p. 2020004, 2021.

[4] Y. Sun, J. Jia, J. Xu, M. Chen, and J. Niu, "Path, feedrate and trajectory planning for free-form surface machining: a stateof-the-art review," Chinese Journal of Aeronautics, 2021.

[5] A. Gameros, S. Lowth, D. Axinte, A. Nagy-Sochacki, O. Craig, and H. R. Siller, "State-of-the-art in fixture systems for the manufacture and assembly of rigid components: a review," 
International Journal of Machine Tools and Manufacture, vol. 123, pp. 1-21, 2017.

[6] B. Li and S. N. Melkote, "Fixture clamping force optimisation and its impact on workpiece location accuracy," The International Journal of Advanced Manufacturing Technology, vol. 17, no. 2, pp. 104-113, 2001.

[7] G. H. Qin, W. H. Zhang, and M. Wan, "A mathematical approach to analysis and optimal design of a fixture locating scheme," International Journal of Advanced Manufacturing Technology, vol. 29, no. 3-4, pp. 349-359, 2006.

[8] M. Sága, M. Blatnická, M. Blatnický, J. Dižo, and J. Gerlici, "Research of the fatigue life of welded joints of high strength steel S960 Q1 created using laser and electron beams," Materials, vol. 13, no. 11, p. 2539, 2020.

[9] M. Estrems, H. T. Sanchez, and F. Faura, "Influence of fixtures on dimensional accuracy in machining processes," The International Journal of Advanced Manufacturing Technology, vol. 21, no. 5, pp. 384-390, 2003.

[10] M. Blatnicky, D. Jan, M. Saga, J. Gerlici, and E. Kuba, "Design of a mechanical part of an automated platform for oblique manipulation," Applied Sciences-Basel, vol. 10, no. 23, p. 8467, 2020.

[11] S. P. Siebenaler and S. N. Melkote, "Prediction of workpiece deformation in a fixture system using the finite element method," International Journal of Machine Tools and Manufacture, vol. 46, no. 1, pp. 51-58, 2006.

[12] A. Raghu and S. N. Melkote, "Analysis of the effects of fixture clamping sequence on part location errors," International Journal of Machine Tools and Manufacture, vol. 44, no. 4, pp. 373-382, 2004.

[13] L. Nagendran, K. Srinivasan, and V. Ranjeev, "Analytical and experimental studies on fixtured work pieces," AIP Conference Proceedings, vol. 712, pp. 690-694, 2004.

[14] M. J. Nategh and H. Parvaz, "Development of computer aided clamping system design for workpieces with freeform surfaces," Computer-Aided Design, vol. 95, pp. 52-61, 2018.

[15] R. Haymanali, E. C. De Meter, and M. W. Trethewey, "Development of a compliance tester for assessing and reducing the static compliance of fixture-workpiece systems," Journal of Manufacturing Systems, vol. 19, no. 2, pp. 108-120, 2000.

[16] E. C. De Meter, W. Xie, S. Choudhuri, S. Vallapuzha, and M. W. Trethewey, "A model to predict minimum required clamp pre-loads in light of fixture-workpiece compliance," International Journal of Machine Tools and Manufacture, vol. 41, no. 7, pp. 1031-1054, 2001.

[17] H. T. Sánchez, M. Estrems, and F. Faura, "Fixturing analysis methods for calculating the contact load distribution and the valid clamping regions in machining processes," The International Journal of Advanced Manufacturing Technology, vol. 29, no. 5, pp. 426-435, 2006.

[18] S. Ratchev, K. Phuah, and S. Liu, "Fea-based methodology for the prediction of part-fixture behaviour and its applications," Journal of Materials Processing Technology, vol. 191, no. 1-3, pp. 260-264, 2007.

[19] B. Li and S. N. Melkote, "Optimal fixture design accounting for the effect of workpiece dynamics," International Journal of Advanced Manufacturing Technology, vol. 18, no. 10, pp. 701-707, 2001.

[20] S. Satyanarayana and S. N. Melkote, "Finite element modeling of fixture-workpiece contacts: single contact modeling and experimental verification," International Journal of Machine Tools and Manufacture, vol. 44, no. 9, pp. 903-913, 2004.

[21] G. Chen, X. Shu, J. Liu, B. Zhang, and J. Feng, "A new method for distortion calculations in additive manufacturing: contact analysis between a workpiece and clamps," International Journal of Mechanical Sciences, vol. 171, p. 105362, 2020.

[22] A. P. Christoforou, A. S. Yigit, and M. Majeed, "Low-velocity impact response of structures with local plastic deformation: characterization and scaling," Journal of Computational and Nonlinear Dynamics, vol. 8, p. 0110121, 2013.

[23] H. Ghaednia, D. B. Marghitu, and R. L. Jackson, "Predicting the permanent deformation after the impact of a rod with a flat surface," Journal of Tribology-Transactions of the ASME, vol. 137, p. 0114031, 2015.

[24] D. Rathbone, M. Marigo, D. Dini, and B. van Wachem, "An accurate force-displacement law for the modelling of elasticplastic contacts in discrete element simulations," Powder Technology, vol. 282, no. SI, pp. 2-9, 2015.

[25] J.-R. Riba, A.-G. Mancini, C. Abomailek, and F. Capelli, “A 3D-fem-based model to predict the electrical constriction resistance of compressed contacts," Measurement, vol. 114, pp. 44-50, 2018.

[26] A. R. Khoei and M. Nikbakht, "Contact friction modeling with the extended finite element method (X-Fem)," Journal of Materials Processing Technology, vol. 177, no. 1-3, pp. 58-62, 2006.

[27] B. Wiegert, H. Hetzler, and W. Seemann, "A simplified elastohydrodynamic contact model capturing the nonlinear vibration behaviour," Tribology International, vol. 59, pp. 79-89, 2013.

[28] B. Hu, C. Zhou, and S. Chen, "Elastic dynamics modelling and analysis for a valve train including oil film stiffness and dry contact stiffness," Mechanism and Machine Theory, vol. 131, pp. 33-47, 2019.

[29] X. D. Lu, J. Zhao, J. L. Mo, Q. Zhang, X. Zhang, and Z. R. Zhou, "Improvement of dynamical and tribological properties of friction systems by introducing parallel-grooved structures in elastic damping components," Composite Structures, vol. 192, pp. 8-19, 2018.

[30] Y. J. G. Liao and S. J. Hu, "Flexible multibody dynamics based fixture-workpiece analysis model for fixturing stability," International Journal of Machine Tools and Manufacture, vol. 40, no. 3, pp. 343-362, 2000.

[31] A. Matsubara, Y. Taniyama, J. Wang, and D. Kono, "Design of a support system with a pivot mechanism for suppressing vibrations in thin-wall milling," CIRP Annals, vol. 66, no. 1, pp. 381-384, 2017.

[32] J. Fei, B. Lin, J. Xiao et al., "Investigation of moving fixture on deformation suppression during milling process of thinwalled structures," Journal of Manufacturing Processes, vol. 32, pp. 403-411, 2018.

[33] J. Ma, D. Zhang, B. Wu, M. Luo, and Y. Liu, "Stability improvement and vibration suppression of the thin-walled workpiece in milling process via magnetorheological fluid flexible fixture," International Journal of Advanced Manufacturing Technology, vol. 88, no. 5-8, pp. 1231-1242, 2017.

[34] M. Meshreki, H. Attia, and J. Koevecses, "A new analytical formulation for the dynamics of multipocket thin-walled structures considering the fixture constraints," Journal of Manufacturing Science and Engineering-Transactions of the ASME, vol. 133, p. 0210142, 2011.

[35] J. Yan and G. Qiang, "Simulation of multi-axis grinding considering runout based on envelope theory," Chinese Journal of Aeronautics, vol. 33, pp. 3526-3534, 2020.

[36] X. Xie, H. Zheng, S. Jonckheere, A. van de Walle, B. Pluymers, and W. Desmet, "Adaptive model reduction technique for large-scale dynamical systems with frequency-dependent 
damping," Computer Methods in Applied Mechanics and Engineering, vol. 332, pp. 363-381, 2018.

[37] M. Meshreki, H. Attia, and J. Koevecses, "Development of a new model for the varying dynamics of flexible pocketstructures during machining," Journal of Manufacturing Science and Engineering-Transactions of the ASME, vol. 133, p. 0410024, 2011.

[38] X.-J. Wan and Y. Zhang, "A novel approach to fixture layout optimization on maximizing dynamic machinability," International Journal of Machine Tools and Manufacture, vol. 70, pp. 32-44, 2013.

[39] F. Pellicano, "Vibrations of circular cylindrical shells: theory and experiments," Journal of Sound and Vibration, vol. 303, no. 1-2, pp. 154-170, 2007.

[40] Z. Luo, N. Sun, Y. Wang, K. Zhang, and Q. Han, "Study of vibration characteristics of the short thin cylindrical shells and its experiment," Journal of Vibroengineering, vol. 15, no. 3, pp. 1270-1283, 2013.

[41] Y. Kurylov and M. Amabili, "Polynomial versus trigonometric expansions for nonlinear vibrations of circular cylindrical shells with different boundary conditions," Journal of Sound and Vibration, vol. 329, no. 9, pp. 1435-1449, 2010.

[42] W. Soedel, Vibrations of Shells and Plates, Marcel Dekker Inc, New York, NY, USA, 2004.

[43] C. T. Loy, K. Y. Lam, and C. Shu, "Analysis of cylindrical shells using generalized differential quadrature," Shock and Vibration, vol. 4, no. 3, pp. 193-198, 1997.

[44] X.-J. Wan, Y. Zhang, and X.-D. Huang, "Investigation of influence of fixture layout on dynamic response of thin-wall multi-framed work-piece in machining," International Journal of Machine Tools and Manufacture, vol. 75, pp. 87-99, 2013.

[45] M. Amabili, Nonlinear Vibrations and Stability of Shells and Plates, Cambridge University Press, New York, NY, USA, 2008.

[46] J. Ma, D. Zhang, B. Wu, M. Luo, and B. Chen, "Vibration suppression of thin-walled workpiece machining considering external damping properties based on magnetorheological fluids flexible fixture," Chinese Journal of Aeronautics, vol. 29, no. 4, pp. 1074-1083, 2016.

[47] D. J. Ewins, Modal Testing: Theory, Practice and Application, Imperial College of Science, Technology and Medicine, London, UK, 2000.

[48] T. A. Harris and M. N. Kotzalas, Essential Concepts of Bearing Technology, Taylor \& Francis, Oxfordshire, UK, 2006.

[49] D. E. Brewe and B. J. Hamrock, "Simplified solution for elliptical-contact deformation between two elastic solids," Journal of Lubrication Technology, vol. 99, no. 4, pp. 485-487, 1977. 